Article

\title{
Hyperfuzzy Ideals in BCK/BCI-Algebras ${ }^{\dagger}$
}

\author{
Seok-Zun Song ${ }^{1}$ (D) , Seon Jeong $\mathrm{Kim}^{2}$ and Young Bae Jun ${ }^{3, *}$ \\ 1 Department of Mathematics, Jeju National University, Jeju 63243, Korea; szsong@jejunu.ac.kr \\ 2 Department of Mathematics, Natural Science of College, Gyeongsang National University, \\ Jinju 52828, Korea; skim@gnu.ac.kr \\ 3 Department of Mathematics Education, Gyeongsang National University, Jinju 52828, Korea \\ * Correspondence: skywine@gmail.com \\ + To the memory of Professor Lotfi A. Zadeh.
}

Received: 17 November 2017; Accepted: 10 December 2017; Published: 14 December 2017

\begin{abstract}
The notions of hyperfuzzy ideals in $B C K / B C I$-algebras are introduced, and related properties are investigated. Characterizations of hyperfuzzy ideals are established. Relations between hyperfuzzy ideals and hyperfuzzy subalgebras are discussed. Conditions for hyperfuzzy subalgebras to be hyperfuzzy ideals are provided.
\end{abstract}

Keywords: hyperfuzzy set; hyperfuzzy subalgebra; hyperfuzzy ideal

\section{Introduction}

After Zadeh [1] has introduced the fundamental concept of fuzzy sets, several generalizations of fuzzy sets are achieved. As a generalization of fuzzy sets and interval-valued fuzzy sets, Ghosh and Samanta [2] introduced the notion of hyperfuzzy sets, and then they applied it to group theory. They defined hyperfuzzy (normal) subgroups and hyperfuzzy cosets, and investigated their properties. The hyperfuzzy set has a subset of the interval $[0,1]$ as its image. Hence, it is a generalization of an interval-valued fuzzy set. In mathematics, $B C K$ and $B C I$-algebras are algebraic structures, introduced by Imai, Iseki and Tanaka, that describe fragments of the propositional calculus involving implication known as $B C K$ and $B C I$ logics (see [3-5]). Jun et al. [6] applied hyperfuzzy sets to $B C K / B C I$-algebras by using the infimum and supremum of the image of hyperfuzzy sets. They introduced the notion of $k$-fuzzy substructure for $k \in\{1,2,3,4\}$ and then they introduced the concepts of hyperfuzzy substructures of several types by using $k$-fuzzy substructures, and investigated their basic properties. They also introduced the notion of hyperfuzzy subalgebras of type $(i, j)$ for $i, j \in\{1,2,3,4\}$, and discussed relations between hyperfuzzy substructure/subalgebra and its length. They investigated the properties of hyperfuzzy subalgebras related to upper and lower level subsets.

The aim of this paper is to study $B C K / B C I$-algebraic structures based on hyperfuzzy structures. So, the notions and results in this manuscript are a generalization of $B C K / B C I$-algebraic structures based on fuzzy and interval-valued fuzzy structures. We introduce the notion of hyperfuzzy ideals in $B C K / B C I$-algebras, and investigate several properties. We consider characterizations of hyperfuzzy ideals, and discuss relations between hyperfuzzy subalgebras and hyperfuzzy ideals. We provide conditions for hyperfuzzy subalgebras to be hyperfuzzy ideals.

\section{Preliminaries}

By a $B C I$-algebra (see $[7,8])$ we mean a system $X:=(X, *, 0)$ in which the following axioms hold:

(I) $\quad((x * y) *(x * z)) *(z * y)=0$,

(II) $(x *(x * y)) * y=0$,

(III) $x * x=0$,

(IV) $x * y=y * x=0 \Rightarrow x=y$ 
for all $x, y, z \in X$. If a $B C I$-algebra $X$ satisfies $0 * x=0$ for all $x \in X$, then we say that $X$ is a $B C K$-algebra $($ see $[7,8])$. We can define a partial ordering $\leq$ by

$$
(\forall x, y \in X)(x \leq y \Longleftrightarrow x * y=0) .
$$

In a $B C K / B C I$-algebra $X$, the following hold (see $[7,8])$ :

$$
\begin{aligned}
& (\forall x \in X)(x * 0=x), \\
& (\forall x, y, z \in X)((x * y) * z=(x * z) * y) .
\end{aligned}
$$

A non-empty subset $S$ of a $B C K / B C I$-algebra $X$ is called a subalgebra of $X($ see $[7,8])$ if $x * y \in S$ for all $x, y \in S$.

We refer the reader to the books $[7,8]$ for further information regarding $B C K / B C I$-algebras.

By a fuzzy structure over a nonempty set $X$ we mean an ordered pair $(X, \rho)$ of $X$ and a fuzzy set $\rho$ on $X$.

Let $X$ be a nonempty set. A mapping $\tilde{\mu}: X \rightarrow \tilde{\mathcal{P}}([0,1])$ is called a hyperfuzzy set over $X$ (see [2]), where $\tilde{\mathcal{P}}([0,1])$ is the family of all nonempty subsets of $[0,1]$. An ordered pair $(X, \tilde{\mu})$ is called a hyper structure over $X$.

Given a hyper structure $(X, \tilde{\mu})$ over a nonempty set $X$, we consider two fuzzy structures $\left(X, \tilde{\mu}_{\text {inf }}\right)$ and $\left(X, \tilde{\mu}_{\text {sup }}\right)$ over $X$ (see [6]) in which

$$
\begin{aligned}
& \tilde{\mu}_{\text {inf }}: X \rightarrow[0,1], \quad x \mapsto \inf \{\tilde{\mu}(x)\}, \\
& \tilde{\mu}_{\text {sup }}: X \rightarrow[0,1], \quad x \mapsto \sup \{\tilde{\mu}(x)\} .
\end{aligned}
$$

Given a nonempty set $X$, let $\mathcal{B}_{K}(X)$ and $\mathcal{B}_{I}(X)$ denote the collection of all $B C K$-algebras and all $B C I$-algebras, respectively. Also $\mathcal{B}(X):=\mathcal{B}_{K}(X) \cup \mathcal{B}_{I}(X)$.

Definition 1 ([6]). For any $(X, *, 0) \in \mathcal{B}(X)$, a fuzzy structure $(X, \mu)$ over $(X, *, 0)$ is called a

- fuzzy subalgebra of $(X, *, 0)$ with type 1 (briefly, 1-fuzzy subalgebra of $(X, *, 0)$ ) if

$$
(\forall x, y \in X)(\mu(x * y) \geq \min \{\mu(x), \mu(y)\}),
$$

- $\quad f u z z y$ subalgebra of $(X, *, 0)$ with type 2 (briefly, 2-fuzzy subalgebra of $(X, *, 0)$ ) if

$$
(\forall x, y \in X)(\mu(x * y) \leq \min \{\mu(x), \mu(y)\}),
$$

- fuzzy subalgebra of $(X, *, 0)$ with type 3 (briefly, 3-fuzzy subalgebra of $(X, *, 0)$ ) if

$$
(\forall x, y \in X)(\mu(x * y) \geq \max \{\mu(x), \mu(y)\}),
$$

- fuzzy subalgebra of $(X, *, 0)$ with type 4 (briefly, 4-fuzzy subalgebra of $(X, *, 0)$ ) if

$$
(\forall x, y \in X)(\mu(x * y) \leq \max \{\mu(x), \mu(y)\}) .
$$

It is clear that every 3-fuzzy subalgebra is a 1-fuzzy subalgebra and every 2-fuzzy subalgebra is a 4-fuzzy subalgebra.

Definition 2 ([6]). For any $(X, *, 0) \in \mathcal{B}(X)$ and $i, j \in\{1,2,3,4\}$, a hyper structure $(X, \tilde{\mu})$ over $(X, *, 0)$ is called an $(i, j)$-hyperfuzzy subalgebra of $(X, *, 0)$ if $\left(X, \tilde{\mu}_{\text {inf }}\right)$ is an $i$-fuzzy subalgebra of $(X, *, 0)$ and $\left(X, \tilde{\mu}_{\text {sup }}\right)$ is a j-fuzzy subalgebra of $(X, *, 0)$. 


\section{Hyperfuzzy Ideals}

In what follows, let $(X, *, 0) \in \mathcal{B}(X)$ unless otherwise specified.

Definition 3. A fuzzy structure $(X, \mu)$ over $(X, *, 0)$ is called a

- $\quad$ fuzzy ideal of $(X, *, 0)$ with type 1 (briefly, 1-fuzzy ideal of $(X, *, 0)$ ) if

$$
\begin{aligned}
& (\forall x \in X)(\mu(0) \geq \mu(x)), \\
& (\forall x, y \in X)(\mu(x) \geq \min \{\mu(x * y), \mu(y)\})
\end{aligned}
$$

- $\quad$ fuzzy ideal of $(X, *, 0)$ with type 2 (briefly, 2-fuzzy ideal of $(X, *, 0)$ ) if

$$
\begin{aligned}
& (\forall x \in X)(\mu(0) \leq \mu(x)) \\
& (\forall x, y \in X)(\mu(x) \leq \min \{\mu(x * y), \mu(y)\})
\end{aligned}
$$

- $\quad$ fuzzy ideal of $(X, *, 0)$ with type 3 (briefly, 3-fuzzy ideal of $(X, *, 0)$ ) if it satisfies $(7)$ and

$$
(\forall x, y \in X)(\mu(x) \geq \max \{\mu(x * y), \mu(y)\})
$$

- $\quad f u z z y$ ideal of $(X, *, 0)$ with type 4 (briefly, 4-fuzzy ideal of $(X, *, 0)$ ) if it satisfies (9) and

$$
(\forall x, y \in X)(\mu(x) \leq \max \{\mu(x * y), \mu(y)\}) .
$$

It is clear that every 3-fuzzy ideal is a 1-fuzzy ideal and every 2-fuzzy ideal is a 4-fuzzy ideal.

Definition 4. For any $i, j \in\{1,2,3,4\}$, a hyper structure $(X, \tilde{\mu})$ over $(X, *, 0)$ is called an $(i, j)$-hyperfuzzy ideal of $(X, *, 0)$ if $\left(X, \tilde{\mu}_{\text {inf }}\right)$ is an $i$-fuzzy ideal of $(X, *, 0)$ and $\left(X, \tilde{\mu}_{\text {sup }}\right)$ is a j-fuzzy ideal of $(X, *, 0)$.

Example 1. Consider a BCK-algebra $X=\{0,1,2,3,4\}$ with the binary operation $*$ which is given in Table 1 (see [8]).

Table 1. Cayley table for the binary operation " $*$ ".

\begin{tabular}{llllll}
\hline$*$ & $\mathbf{0}$ & $\mathbf{1}$ & $\mathbf{2}$ & $\mathbf{3}$ & $\mathbf{4}$ \\
\hline 0 & 0 & 0 & 0 & 0 & 0 \\
1 & 1 & 0 & 1 & 0 & 0 \\
2 & 2 & 2 & 0 & 0 & 0 \\
3 & 3 & 3 & 3 & 0 & 0 \\
4 & 4 & 3 & 4 & 1 & 0 \\
\hline
\end{tabular}

(1) Let $(X, \tilde{\mu})$ be a hyper structure over $(X, *, 0)$ in which $\tilde{\mu}$ is given as follows:

$$
\tilde{\mu}: X \rightarrow \tilde{\mathcal{P}}([0,1]), x \mapsto \begin{cases}{[0.5,0.6)} & \text { if } x=0, \\ {[0.4,0.8]} & \text { if } x=1, \\ {[0.3,0.7]} & \text { if } x=2, \\ {[0.2,0.9]} & \text { if } x \in\{3,4\} .\end{cases}
$$

It is routine to verify that $(X, \tilde{\mu})$ is a $(1,4)$-hyperfuzzy ideal of $(X, *, 0)$. 
(2) Let $(X, \tilde{\mu})$ be a hyper structure over $(X, *, 0)$ in which $\tilde{\mu}$ is given as follows:

$$
\tilde{\mu}: X \rightarrow \tilde{\mathcal{P}}([0,1]), x \mapsto \begin{cases}{[0.5,0.9)} & \text { if } x=0, \\ (0.4,0.9] & \text { if } x=1, \\ {[0.3,0.5]} & \text { if } x=2, \\ {[0.2,0.4]} & \text { if } x \in\{3,4\} .\end{cases}
$$

It is routine to verify that $(X, \tilde{\mu})$ is a $(1,1)$-hyperfuzzy ideal of $(X, *, 0)$.

Example 2. Consider a BCI-algebra $X=\{0,1,2, a, b\}$ with the binary operation $*$ which is given in Table 2 (see [8]).

Table 2. Cayley table for the binary operation " $*$ ".

\begin{tabular}{llllll}
\hline$*$ & $\mathbf{0}$ & $\mathbf{1}$ & $\mathbf{2}$ & $\boldsymbol{a}$ & $\boldsymbol{b}$ \\
\hline 0 & 0 & 0 & 0 & $a$ & $a$ \\
1 & 1 & 0 & 1 & $b$ & $a$ \\
2 & 2 & 2 & 0 & $a$ & $a$ \\
$a$ & $a$ & $a$ & $a$ & 0 & 0 \\
$b$ & $b$ & $a$ & $b$ & 1 & 0 \\
\hline
\end{tabular}

(1) Let $(X, \tilde{\mu})$ be a hyper structure over $(X, *, 0)$ in which $\tilde{\mu}$ is given as follows:

$$
\tilde{\mu}: X \rightarrow \tilde{\mathcal{P}}([0,1]), x \mapsto \begin{cases}{[0.33,0.75)} & \text { if } x=0, \\ {[0.63,0.75]} & \text { if } x=1, \\ {[0.43,0.70]} & \text { if } x=2, \\ {[0.53,0.65]} & \text { if } x=a, \\ {[0.63,0.65]} & \text { if } x=b .\end{cases}
$$

By routine calculations, we know that $(X, \tilde{\mu})$ is a $(4,1)$-hyperfuzzy ideal of $(X, *, 0)$.

(2) Let $(X, \tilde{\mu})$ be a hyper structure over $(X, *, 0)$ in which $\tilde{\mu}$ is given as follows:

$$
\tilde{\mu}: X \rightarrow \tilde{\mathcal{P}}([0,1]), x \mapsto \begin{cases}{[0.33,0.39)} & \text { if } x=0, \\ {[0.63,0.69]} & \text { if } x=1, \\ {[0.43,0.49]} & \text { if } x=2, \\ {[0.53,0.59]} & \text { if } x=a, \\ {[0.63,0.69]} & \text { if } x=b .\end{cases}
$$

By routine calculations, we know that $(X, \tilde{\mu})$ is a $(4,4)$-hyperfuzzy ideal of $(X, *, 0)$.

Proposition 1. Given a hyper structure $(X, \tilde{\mu})$ over $(X, *, 0)$, we have the following assertions.

(1) If $(X, \tilde{\mu})$ is a $(1,4)$-hyperfuzzy ideal of $(X, *, 0)$, then

$$
(\forall x, y \in X)\left(x \leq y \Rightarrow \tilde{\mu}_{\text {inf }}(x) \geq \tilde{\mu}_{\text {inf }}(y), \tilde{\mu}_{\text {sup }}(x) \leq \tilde{\mu}_{\text {sup }}(y)\right) .
$$

(2) If $(X, \tilde{\mu})$ is a $(1,1)$-hyperfuzzy ideal of $(X, *, 0)$, then

$$
(\forall x, y \in X)\left(x \leq y \Rightarrow \tilde{\mu}_{\text {inf }}(x) \geq \tilde{\mu}_{\text {inf }}(y), \tilde{\mu}_{\text {sup }}(x) \geq \tilde{\mu}_{\text {sup }}(y)\right) .
$$

(3) If $(X, \tilde{\mu})$ is a $(4,1)$-hyperfuzzy ideal of $(X, *, 0)$, then

$$
(\forall x, y \in X)\left(x \leq y \Rightarrow \tilde{\mu}_{\text {inf }}(x) \leq \tilde{\mu}_{\text {inf }}(y), \tilde{\mu}_{\text {sup }}(x) \geq \tilde{\mu}_{\text {sup }}(y)\right) .
$$


(4) If $(X, \tilde{\mu})$ is a $(4,4)$-hyperfuzzy ideal of $(X, *, 0)$, then

$$
(\forall x, y \in X)\left(x \leq y \Rightarrow \tilde{\mu}_{\text {inf }}(x) \leq \tilde{\mu}_{\text {inf }}(y), \tilde{\mu}_{\text {sup }}(x) \leq \tilde{\mu}_{\text {sup }}(y)\right)
$$

Proof. If $(X, \tilde{\mu})$ is a $(1,4)$-hyperfuzzy ideal of $(X, *, 0)$, then $\left(X, \tilde{\mu}_{\text {inf }}\right)$ is a 1-fuzzy ideal of $(X, *, 0)$ and $\left(X, \tilde{\mu}_{\text {sup }}\right)$ is a 4 -fuzzy ideal of $(X, *, 0)$. Let $x, y \in X$ be such that $x \leq y$. Then $x * y=0$, and so

$$
\tilde{\mu}_{\text {inf }}(x) \geq \min \left\{\tilde{\mu}_{\text {inf }}(x * y), \tilde{\mu}_{\text {inf }}(y)\right\}=\min \left\{\tilde{\mu}_{\text {inf }}(0), \tilde{\mu}_{\text {inf }}(y)\right\}=\tilde{\mu}_{\text {inf }}(y)
$$

by (8) and (7), and

$$
\tilde{\mu}_{\text {sup }}(x) \leq \max \left\{\tilde{\mu}_{\text {sup }}(x * y), \tilde{\mu}_{\text {sup }}(y)\right\}=\max \left\{\tilde{\mu}_{\text {sup }}(0), \tilde{\mu}_{\text {sup }}(y)\right\}=\tilde{\mu}_{\text {sup }}(y)
$$

by (12) and (9). Similarly, we can prove (2), (3) and (4).

Proposition 2. Given a hyper structure $(X, \tilde{\mu})$ over $(X, *, 0)$, we have the following assertions.

(1) If $(X, \tilde{\mu})$ is an $(i, j)$-hyperfuzzy ideal of $(X, *, 0)$ for $(i, j) \in\{(2,2),(2,3),(3,2),(3,3)\}$, then

$$
(\forall x, y \in X)\left(x \leq y \Rightarrow \tilde{\mu}_{\mathrm{inf}}(x)=\tilde{\mu}_{\mathrm{inf}}(0), \tilde{\mu}_{\mathrm{sup}}(x)=\tilde{\mu}_{\mathrm{sup}}(0)\right)
$$

(2) If $(X, \tilde{\mu})$ is either a $(1,2)$-hyperfuzzy ideal or a $(1,3)$-hyperfuzzy ideal of $(X, *, 0)$, then the following assertion is valid.

$$
(\forall x, y \in X)\left(x \leq y \Rightarrow \tilde{\mu}_{\text {inf }}(x) \geq \tilde{\mu}_{\text {inf }}(y), \tilde{\mu}_{\text {sup }}(x)=\tilde{\mu}_{\text {sup }}(0)\right)
$$

(3) If $(X, \tilde{\mu})$ is either a $(2,1)$-hyperfuzzy ideal or a $(3,1)$-hyperfuzzy ideal of $(X, *, 0)$, then the following assertion is valid.

$$
(\forall x, y \in X)\left(x \leq y \Rightarrow \tilde{\mu}_{\mathrm{inf}}(x)=\tilde{\mu}_{\mathrm{inf}}(0), \tilde{\mu}_{\mathrm{sup}}(x) \geq \tilde{\mu}_{\mathrm{sup}}(y)\right)
$$

(4) If $(X, \tilde{\mu})$ is either a $(2,4)$-hyperfuzzy ideal or a $(3,4)$-hyperfuzzy ideal of $(X, *, 0)$, then the following assertion is valid.

$$
(\forall x, y \in X)\left(x \leq y \Rightarrow \tilde{\mu}_{\text {inf }}(x)=\tilde{\mu}_{\text {inf }}(0), \tilde{\mu}_{\text {sup }}(x) \leq \tilde{\mu}_{\text {sup }}(y)\right)
$$

(5) If $(X, \tilde{\mu})$ is either a $(4,2)$-hyperfuzzy ideal or a $(4,3)$-hyperfuzzy ideal of $(X, *, 0)$, then the following assertion is valid.

$$
(\forall x, y \in X)\left(x \leq y \Rightarrow \tilde{\mu}_{\text {inf }}(x) \leq \tilde{\mu}_{\text {inf }}(y), \tilde{\mu}_{\text {sup }}(x)=\tilde{\mu}_{\text {sup }}(0)\right)
$$

Proof. We prove (1) only, Others can be verified by the similar way. If $(X, \tilde{\mu})$ is a $(2,3)$-hyperfuzzy ideal of $(X, *, 0)$, then $\left(X, \tilde{\mu}_{\text {inf }}\right)$ is a 2-fuzzy ideal of $(X, *, 0)$ and $\left(X, \tilde{\mu}_{\text {sup }}\right)$ is a 3 -fuzzy ideal of $(X, *, 0)$. Let $x, y \in X$ be such that $x \leq y$. Then $x * y=0$, and thus

$$
\begin{aligned}
& \tilde{\mu}_{\text {inf }}(x) \leq \min \left\{\tilde{\mu}_{\text {inf }}(x * y), \tilde{\mu}_{\text {inf }}(y)\right\}=\min \left\{\tilde{\mu}_{\text {inf }}(0), \tilde{\mu}_{\text {inf }}(y)\right\}=\tilde{\mu}_{\text {inf }}(0) \\
& \tilde{\mu}_{\text {sup }}(x) \geq \max \left\{\tilde{\mu}_{\text {sup }}(x * y), \tilde{\mu}_{\text {sup }}(y)\right\}=\max \left\{\tilde{\mu}_{\text {sup }}(0), \tilde{\mu}_{\text {sup }}(y)\right\}=\tilde{\mu}_{\text {sup }}(0)
\end{aligned}
$$

by (10), (9), (11) and (7). Since $\tilde{\mu}_{\text {inf }}(0) \leq \tilde{\mu}_{\text {inf }}(x)$ and $\tilde{\mu}_{\text {sup }}(0) \geq \tilde{\mu}_{\text {sup }}(x)$ for all $x \in X$, it follows that $\tilde{\mu}_{\text {inf }}(0)=\tilde{\mu}_{\text {inf }}(x)$ and $\tilde{\mu}_{\text {sup }}(0)=\tilde{\mu}_{\text {sup }}(x)$ for all $x \in X$. Similarly, we can verify that (17) is true for $(i, j) \in\{(2,2),(3,2),(3,3)\}$.

Proposition 3. Given a hyper structure $(X, \tilde{\mu})$ over $(X, *, 0)$, we have the following assertions. 
(1) If $(X, \tilde{\mu})$ is a $(1,4)$-hyperfuzzy ideal of $(X, *, 0)$, then

$$
(\forall x, y, z \in X)\left(x * y \leq z \Rightarrow\left\{\begin{array}{l}
\tilde{\mu}_{\text {inf }}(x) \geq \min \left\{\tilde{\mu}_{\text {inf }}(y), \tilde{\mu}_{\text {inf }}(z)\right\} \\
\tilde{\mu}_{\text {sup }}(x) \leq \max \left\{\tilde{\mu}_{\text {sup }}(y), \tilde{\mu}_{\text {sup }}(z)\right\}
\end{array}\right) .\right.
$$

(2) If $(X, \tilde{\mu})$ is a $(1,1)$-hyperfuzzy ideal of $(X, *, 0)$, then

$$
(\forall x, y, z \in X)\left(x * y \leq z \Rightarrow\left\{\begin{array}{l}
\tilde{\mu}_{\text {inf }}(x) \geq \min \left\{\tilde{\mu}_{\text {inf }}(y), \tilde{\mu}_{\text {inf }}(z)\right\} \\
\tilde{\mu}_{\text {sup }}(x) \geq \min \left\{\tilde{\mu}_{\text {sup }}(y), \tilde{\mu}_{\text {sup }}(z)\right\}
\end{array}\right) .\right.
$$

(3) If $(X, \tilde{\mu})$ is a $(4,1)$-hyperfuzzy ideal of $(X, *, 0)$, then

$$
(\forall x, y, z \in X)\left(x * y \leq z \Rightarrow\left\{\begin{array}{l}
\tilde{\mu}_{\text {inf }}(x) \leq \max \left\{\tilde{\mu}_{\text {inf }}(y), \tilde{\mu}_{\text {inf }}(z)\right\} \\
\tilde{\mu}_{\text {sup }}(x) \geq \min \left\{\tilde{\mu}_{\text {sup }}(y), \tilde{\mu}_{\text {sup }}(z)\right\}
\end{array}\right) .\right.
$$

(4) If $(X, \tilde{\mu})$ is a $(4,4)$-hyperfuzzy ideal of $(X, *, 0)$, then

$$
(\forall x, y, z \in X)\left(x * y \leq z \Rightarrow\left\{\begin{array}{l}
\tilde{\mu}_{\text {inf }}(x) \leq \max \left\{\tilde{\mu}_{\text {inf }}(y), \tilde{\mu}_{\text {inf }}(z)\right\} \\
\tilde{\mu}_{\text {sup }}(x) \leq \max \left\{\tilde{\mu}_{\text {sup }}(y), \tilde{\mu}_{\text {sup }}(z)\right\}
\end{array}\right) .\right.
$$

Proof. Assume that $(X, \tilde{\mu})$ is a $(1,4)$-hyperfuzzy ideal of $(X, *, 0)$. Let $x, y, z \in X$ be such that $x * y \leq z$. Then $(x * y) * z=0$, and so

$$
\begin{aligned}
\tilde{\mu}_{\mathrm{inf}}(x) & \geq \min \left\{\tilde{\mu}_{\mathrm{inf}}(x * y), \tilde{\mu}_{\mathrm{inf}}(y)\right\} \\
& \geq \min \left\{\min \left\{\tilde{\mu}_{\mathrm{inf}}((x * y) * z), \tilde{\mu}_{\mathrm{inf}}(z)\right\}, \tilde{\mu}_{\mathrm{inf}}(y)\right\} \\
& =\min \left\{\min \left\{\tilde{\mu}_{\mathrm{inf}}(0), \tilde{\mu}_{\mathrm{inf}}(z)\right\}, \tilde{\mu}_{\mathrm{inf}}(y)\right\} \\
& =\min \left\{\tilde{\mu}_{\mathrm{inf}}(y), \tilde{\mu}_{\mathrm{inf}}(z)\right\}
\end{aligned}
$$

by (8) and (7), and

$$
\begin{aligned}
\tilde{\mu}_{\text {sup }}(x) & \leq \max \left\{\tilde{\mu}_{\text {sup }}(x * y), \tilde{\mu}_{\text {sup }}(y)\right\} \\
& \leq \max \left\{\max \left\{\tilde{\mu}_{\text {sup }}((x * y) * z), \tilde{\mu}_{\text {sup }}(z)\right\}, \tilde{\mu}_{\text {sup }}(y)\right\} \\
& =\max \left\{\max \left\{\tilde{\mu}_{\text {sup }}(0), \tilde{\mu}_{\text {sup }}(z)\right\}, \tilde{\mu}_{\text {sup }}(y)\right\} \\
& =\max \left\{\tilde{\mu}_{\text {sup }}(y), \tilde{\mu}_{\text {sup }}(z)\right\}
\end{aligned}
$$

by (12) and (9). Similarly, we can check that (2), (3) and (4) hold.

Proposition 4. Given a hyper structure $(X, \tilde{\mu})$ over $(X, *, 0)$, we have the following assertions.

(1) If $(X, \tilde{\mu})$ is an $(i, j)$-hyperfuzzy ideal of $(X, *, 0)$ for $(i, j) \in\{(2,2),(2,3),(3,2),(3,3)\}$, then

$$
(\forall x, y, z \in X)\left(x * y \leq z \Rightarrow \tilde{\mu}_{\text {inf }}(x)=\tilde{\mu}_{\text {inf }}(0), \tilde{\mu}_{\text {sup }}(x)=\tilde{\mu}_{\text {sup }}(0)\right)
$$

(2) If $(X, \tilde{\mu})$ is either a $(1,2)$-hyperfuzzy ideal or a $(1,3)$-hyperfuzzy ideal of $(X, *, 0)$, then the following assertion is valid.

$$
(\forall x, y, z \in X)\left(x * y \leq z \Rightarrow\left\{\begin{array}{l}
\tilde{\mu}_{\text {inf }}(x) \geq \min \left\{\tilde{\mu}_{\text {inf }}(y), \tilde{\mu}_{\text {inf }}(z)\right\} \\
\tilde{\mu}_{\text {sup }}(x)=\tilde{\mu}_{\text {sup }}(0)
\end{array}\right) .\right.
$$


(3) If $(X, \tilde{\mu})$ is either a $(2,1)$-hyperfuzzy ideal or a $(3,1)$-hyperfuzzy ideal of $(X, *, 0)$, then the following assertion is valid.

$$
(\forall x, y, z \in X)\left(x * y \leq z \Rightarrow\left\{\begin{array}{l}
\tilde{\mu}_{\text {inf }}(x)=\tilde{\mu}_{\text {inf }}(0) \\
\tilde{\mu}_{\text {sup }}(x) \geq \min \left\{\tilde{\mu}_{\text {sup }}(y), \tilde{\mu}_{\text {sup }}(z)\right\}
\end{array}\right) .\right.
$$

(4) If $(X, \tilde{\mu})$ is either a $(2,4)$-hyperfuzzy ideal or a $(3,4)$-hyperfuzzy ideal of $(X, *, 0)$, then the following assertion is valid.

$$
(\forall x, y, z \in X)\left(x * y \leq z \Rightarrow\left\{\begin{array}{l}
\tilde{\mu}_{\text {inf }}(x)=\tilde{\mu}_{\text {inf }}(0) \\
\tilde{\mu}_{\text {sup }}(x) \leq \max \left\{\tilde{\mu}_{\text {sup }}(y), \tilde{\mu}_{\text {sup }}(z)\right\}
\end{array}\right) .\right.
$$

(5) If $(X, \tilde{\mu})$ is either a $(4,2)$-hyperfuzzy ideal or a $(4,3)$-hyperfuzzy ideal of $(X, *, 0)$, then the following assertion is valid.

$$
(\forall x, y, z \in X)\left(x * y \leq z \Rightarrow\left\{\begin{array}{l}
\tilde{\mu}_{\text {inf }}(x) \leq \max \left\{\tilde{\mu}_{\text {inf }}(y), \tilde{\mu}_{\text {inf }}(z)\right\} \\
\tilde{\mu}_{\text {sup }}(x)=\tilde{\mu}_{\text {sup }}(0)
\end{array}\right) .\right.
$$

Proof. If $(X, \tilde{\mu})$ is a $(2,3)$-hyperfuzzy ideal of $(X, *, 0)$, then $\left(X, \tilde{\mu}_{\text {inf }}\right)$ is a 2-fuzzy ideal of $(X, *, 0)$ and $\left(X, \tilde{\mu}_{\text {sup }}\right)$ is a 3 -fuzzy ideal of $(X, *, 0)$. Let $x, y, z \in X$ be such that $x * y \leq z$. Then $(x * y) * z=0$, and so

$$
\begin{aligned}
\tilde{\mu}_{\mathrm{inf}}(x) & \leq \min \left\{\tilde{\mu}_{\mathrm{inf}}(x * y), \tilde{\mu}_{\mathrm{inf}}(y)\right\} \\
& \leq \min \left\{\min \left\{\tilde{\mu}_{\mathrm{inf}}((x * y) * z), \tilde{\mu}_{\mathrm{inf}}(z)\right\}, \tilde{\mu}_{\mathrm{inf}}(y)\right\} \\
& =\min \left\{\min \left\{\tilde{\mu}_{\mathrm{inf}}(0), \tilde{\mu}_{\mathrm{inf}}(z)\right\}, \tilde{\mu}_{\mathrm{inf}}(y)\right\} \\
& =\min \left\{\tilde{\mu}_{\mathrm{inf}}(0), \tilde{\mu}_{\mathrm{inf}}(y)\right\} \\
& =\tilde{\mu}_{\mathrm{inf}}(0)
\end{aligned}
$$

by (10) and (9), and

$$
\begin{aligned}
\tilde{\mu}_{\text {sup }}(x) & \geq \max \left\{\tilde{\mu}_{\text {sup }}(x * y), \tilde{\mu}_{\text {sup }}(y)\right\} \\
& \geq \max \left\{\max \left\{\tilde{\mu}_{\text {sup }}((x * y) * z), \tilde{\mu}_{\text {sup }}(z)\right\}, \tilde{\mu}_{\text {sup }}(y)\right\} \\
& =\max \left\{\max \left\{\tilde{\mu}_{\text {sup }}(0), \tilde{\mu}_{\text {sup }}(z)\right\}, \tilde{\mu}_{\text {sup }}(y)\right\} \\
& =\max \left\{\tilde{\mu}_{\text {sup }}(0), \tilde{\mu}_{\text {sup }}(y)\right\} \\
& =\tilde{\mu}_{\text {sup }}(0)
\end{aligned}
$$

by (11) and (7). Since $\tilde{\mu}_{\text {inf }}(0) \leq \tilde{\mu}_{\text {inf }}(x)$ and $\tilde{\mu}_{\text {sup }}(0) \geq \tilde{\mu}_{\text {sup }}(x)$ for all $x \in X$, it follows that $\tilde{\mu}_{\text {inf }}(x)=\tilde{\mu}_{\text {inf }}(0)$ and $\tilde{\mu}_{\text {sup }}(x)=\tilde{\mu}_{\text {sup }}(0)$. Similarly, we can verify that $(26)$ is true for $(i, j) \in$ $\{(2,2),(3,2),(3,3)\}$. Similarly, we can show that (2), (3), (4) and (5) are true.

Given a hyper structure $(X, \tilde{\mu})$ over $X$ and $\alpha, \beta \in[0,1]$, we consider the following sets:

$$
\begin{aligned}
& U\left(\tilde{\mu}_{\text {inf }} ; \alpha\right):=\left\{x \in X \mid \tilde{\mu}_{\text {inf }}(x) \geq \alpha\right\}, \\
& L\left(\tilde{\mu}_{\text {inf }} ; \alpha\right):=\left\{x \in X \mid \tilde{\mu}_{\text {inf }}(x) \leq \alpha\right\}, \\
& U\left(\tilde{\mu}_{\text {sup }} ; \beta\right):=\left\{x \in X \mid \tilde{\mu}_{\text {sup }}(x) \geq \beta\right\}, \\
& L\left(\tilde{\mu}_{\text {sup }} ; \beta\right):=\left\{x \in X \mid \tilde{\mu}_{\text {sup }}(x) \leq \beta\right\} .
\end{aligned}
$$

Theorem 1. (1) A hyper structure $(X, \tilde{\mu})$ over $(X, *, 0)$ is a $(1,4)$-hyperfuzzy ideal of $(X, *, 0)$ if and only if the sets $U\left(\tilde{\mu}_{\text {inf }} ; \alpha\right)$ and $L\left(\tilde{\mu}_{\text {sup }} ; \beta\right)$ are either empty or ideals of $X$ for all $\alpha, \beta \in[0,1]$. 
(2) A hyper structure $(X, \tilde{\mu})$ over $(X, *, 0)$ is a $(1,1)$-hyperfuzzy ideal of $(X, *, 0)$ if and only if the sets $U\left(\tilde{\mu}_{\text {inf }} ; \alpha\right)$ and $U\left(\tilde{\mu}_{\text {sup }} ; \beta\right)$ are either empty or ideals of $X$ for all $\alpha, \beta \in[0,1]$.

(3) A hyper structure $(X, \tilde{\mu})$ over $(X, *, 0)$ is a $(4,1)$-hyperfuzzy ideal of $(X, *, 0)$ if and only if the sets $L\left(\tilde{\mu}_{\text {inf }} ; \alpha\right)$ and $U\left(\tilde{\mu}_{\text {sup }} ; \beta\right)$ are either empty or ideals of $X$ for all $\alpha, \beta \in[0,1]$.

(4) A hyper structure $(X, \tilde{\mu})$ over $(X, *, 0)$ is a $(4,4)$-hyperfuzzy ideal of $(X, *, 0)$ if and only if the sets $L\left(\tilde{\mu}_{\text {inf }} ; \alpha\right)$ and $L\left(\tilde{\mu}_{\text {sup }} ; \beta\right)$ are either empty or ideals of $X$ for all $\alpha, \beta \in[0,1]$.

Proof. Assume that $(X, \tilde{\mu})$ is a $(1,4)$-hyperfuzzy ideal of $(X, *, 0)$ and $U\left(\tilde{\mu}_{\text {inf }} ; \alpha\right) \neq \varnothing \neq L\left(\tilde{\mu}_{\text {sup }} ; \beta\right)$ for $\alpha, \beta \in[0,1]$. Then there exist $a \in U\left(\tilde{\mu}_{\text {inf }} ; \alpha\right)$ and $b \in L\left(\tilde{\mu}_{\text {sup }} ; \beta\right)$. Hence $\tilde{\mu}_{\text {inf }}(0) \geq \tilde{\mu}_{\text {inf }}(a) \geq \alpha$ and $\tilde{\mu}_{\text {sup }}(0) \leq \tilde{\mu}_{\text {sup }}(b) \leq \beta$, that is, $0 \in U\left(\tilde{\mu}_{\text {inf }} ; \alpha\right) \cap L\left(\tilde{\mu}_{\text {sup }} ; \beta\right)$. Let $x, y \in X$ be such that $x * y \in U\left(\tilde{\mu}_{\text {inf }} ; \alpha\right)$ and $y \in U\left(\tilde{\mu}_{\text {inf }} ; \alpha\right)$. Then $\tilde{\mu}_{\text {inf }}(x * y) \geq \alpha$ and $\tilde{\mu}_{\text {inf }}(y) \geq \alpha$. It follows that

$$
\tilde{\mu}_{\text {inf }}(x) \geq \min \left\{\tilde{\mu}_{\text {inf }}(x * y), \tilde{\mu}_{\text {inf }}(y)\right\} \geq \alpha
$$

and so that $x \in U\left(\tilde{\mu}_{\text {inf }} ; \alpha\right)$. Thus $U\left(\tilde{\mu}_{\text {inf }} ; \alpha\right)$ is an ideal of $(X, *, 0)$. Now let $a, b \in X$ be such that $a * b \in L\left(\tilde{\mu}_{\text {sup }} ; \beta\right)$ and $b \in L\left(\tilde{\mu}_{\text {sup }} ; \beta\right)$. Then $\tilde{\mu}_{\text {sup }}(a * b) \leq \beta$ and $\tilde{\mu}_{\text {sup }}(b) \leq \beta$, which imply that

$$
\tilde{\mu}_{\text {sup }}(a) \leq \max \left\{\tilde{\mu}_{\text {sup }}(a * b), \tilde{\mu}_{\text {sup }}(b)\right\} \leq \beta .
$$

Thus $a \in L\left(\tilde{\mu}_{\text {sup }} ; \beta\right)$, and therefore $L\left(\tilde{\mu}_{\text {sup }} ; \beta\right)$ is an ideal of $(X, *, 0)$.

Conversely, suppose that the sets $U\left(\tilde{\mu}_{\text {inf }} ; \alpha\right)$ and $L\left(\tilde{\mu}_{\text {sup }} ; \beta\right)$ are either empty or ideals of $X$ for all $\alpha, \beta \in[0,1]$. For any $x \in X$, let $\tilde{\mu}_{\text {inf }}(x)=\alpha$ and $\tilde{\mu}_{\text {sup }}(x)=\beta$. Then $x \in U\left(\tilde{\mu}_{\text {inf }} ; \alpha\right) \cap L\left(\tilde{\mu}_{\text {sup }} ; \beta\right)$, and so $U\left(\tilde{\mu}_{\text {inf }} ; \alpha\right)$ and $L\left(\tilde{\mu}_{\text {sup }} ; \beta\right)$ are nonempty. Hence $U\left(\tilde{\mu}_{\text {inf }} ; \alpha\right)$ and $L\left(\tilde{\mu}_{\text {sup }} ; \beta\right)$ are ideals of $(X, *, 0)$, and thus $0 \in U\left(\tilde{\mu}_{\text {inf }} ; \alpha\right) \cap L\left(\tilde{\mu}_{\text {sup }} ; \beta\right)$. It follows that $\tilde{\mu}_{\text {inf }}(0) \geq \alpha=\tilde{\mu}_{\text {inf }}(x)$ and $\tilde{\mu}_{\text {sup }}(0) \leq \beta=\tilde{\mu}_{\text {sup }}(x)$ for all $x \in X$. Assume that there exist $a, b \in X$ such that

$$
\tilde{\mu}_{\text {inf }}(a)<\min \left\{\tilde{\mu}_{\text {inf }}(a * b), \tilde{\mu}_{\text {inf }}(b)\right\} .
$$

If we take $\gamma:=\min \left\{\tilde{\mu}_{\text {inf }}(a * b), \tilde{\mu}_{\text {inf }}(b)\right\}$, then $\gamma \in[0,1], a * b \in U\left(\tilde{\mu}_{\text {inf }} ; \gamma\right)$ and $b \in U\left(\tilde{\mu}_{\text {inf }} ; \gamma\right)$. Since $U\left(\tilde{\mu}_{\text {inf }} ; \gamma\right)$ is an ideal of $X$, we have $a \in U\left(\tilde{\mu}_{\text {inf }} ; \gamma\right)$, that is, $\tilde{\mu}_{\text {inf }}(a) \geq \gamma$. This is a contradiction, and so

$$
\tilde{\mu}_{\text {inf }}(x) \geq \min \left\{\tilde{\mu}_{\text {inf }}(x * y), \tilde{\mu}_{\text {inf }}(y)\right\}
$$

for all $x, y \in X$. Now, suppose that

$$
\tilde{\mu}_{\text {sup }}(x)>\max \left\{\tilde{\mu}_{\text {sup }}(x * y), \tilde{\mu}_{\text {sup }}(y)\right\}
$$

for some $x, y \in X$, and take

$$
\beta:=\frac{1}{2}\left(\tilde{\mu}_{\text {sup }}(x)+\max \left\{\tilde{\mu}_{\text {sup }}(x * y), \tilde{\mu}_{\text {sup }}(y)\right\}\right) .
$$

Then $x * y \in L\left(\tilde{\mu}_{\text {sup }} ; \beta\right)$ and $y \in L\left(\tilde{\mu}_{\text {sup }} ; \beta\right)$, which imply that $x \in L\left(\tilde{\mu}_{\text {sup }} ; \beta\right)$ since $L\left(\tilde{\mu}_{\text {sup }} ; \beta\right)$ is an ideal of $X$. Hence $\tilde{\mu}_{\text {sup }}(x) \leq \beta$, which is a contradiction, and so

$$
\tilde{\mu}_{\text {sup }}(x) \leq \max \left\{\tilde{\mu}_{\text {sup }}(x * y), \tilde{\mu}_{\text {sup }}(y)\right\}
$$

for all $x, y \in X$. Therefore $(X, \tilde{\mu})$ is a $(1,4)$-hyperfuzzy ideal of $(X, *, 0)$. Similarly, we can verify that (2), (3), and (4) hold.

Theorem 2. If a hyper structure $(X, \tilde{\mu})$ over $(X, *, 0)$ is a $(2,3)$-hyperfuzzy ideal of $(X, *, 0)$, then the sets $U\left(\tilde{\mu}_{\text {inf }} ; \alpha\right)^{c}$ and $L\left(\tilde{\mu}_{\text {sup }} ; \beta\right)^{c}$ are either empty or ideals of $(X, *, 0)$ for all $\alpha, \beta \in[0,1]$. 
Proof. If a hyper structure $(X, \tilde{\mu})$ over $(X, *, 0)$ is a $(2,3)$-hyperfuzzy ideal of $(X, *, 0)$, then $\left(X, \tilde{\mu}_{\text {inf }}\right)$ is a 2-fuzzy ideal of $X$ and $\left(X, \tilde{\mu}_{\text {sup }}\right)$ is a 3-fuzzy ideal of $(X, *, 0)$. Let $\alpha, \beta \in[0,1]$ be such that $U\left(\tilde{\mu}_{\text {inf }} ; \alpha\right)^{c}$ and $L\left(\tilde{\mu}_{\text {sup }} ; \beta\right)^{c}$ are nonempty. Then there exist $x, a \in X$ such that $x \in U\left(\tilde{\mu}_{\text {inf }} ; \alpha\right)^{c}$ and $a \in L\left(\tilde{\mu}_{\text {sup }} ; \beta\right)^{c}$. Hence $\tilde{\mu}_{\text {inf }}(0) \leq \tilde{\mu}_{\text {inf }}(x)<\alpha$ and $\tilde{\mu}_{\text {sup }}(0) \geq \tilde{\mu}_{\text {sup }}(a)>\beta$, which imply that $0 \in U\left(\tilde{\mu}_{\text {inf }} ; \alpha\right)^{c} \cap L\left(\tilde{\mu}_{\text {sup }} ; \beta\right)^{c}$. Let $x, y \in X$ be such that $x * y \in U\left(\tilde{\mu}_{\text {inf }} ; \alpha\right)^{c}$ and $y \in U\left(\tilde{\mu}_{\text {inf }} ; \alpha\right)^{c}$. Then $\tilde{\mu}_{\text {inf }}(x * y)<\alpha$ and $\tilde{\mu}_{\text {inf }}(y)<\alpha$. It follows from (10) that

$$
\tilde{\mu}_{\text {inf }}(x) \leq \min \left\{\tilde{\mu}_{\text {inf }}(x * y), \tilde{\mu}_{\text {inf }}(y)\right\}<\alpha
$$

and so that $x \in U\left(\tilde{\mu}_{\text {inf }} ; \alpha\right)^{c}$. Hence $U\left(\tilde{\mu}_{\text {inf }} ; \alpha\right)^{c}$ is an ideal of $(X, *, 0)$. Now let $a, b \in X$ be such that $a * b \in L\left(\tilde{\mu}_{\text {sup }} ;\right)^{c}$ and $b \in L\left(\tilde{\mu}_{\text {sup }} ; \beta\right)^{c}$. Then $\tilde{\mu}_{\text {sup }}(a * b)>\beta$ and $\tilde{\mu}_{\text {sup }}(b)>\beta$, which imply from (11) that

$$
\tilde{\mu}_{\text {sup }}(a) \geq \max \left\{\tilde{\mu}_{\text {sup }}(a * b), \tilde{\mu}_{\text {sup }}(b)\right\}>\beta
$$

Thus $a \in L\left(\tilde{\mu}_{\text {sup }} ; \beta\right)^{\mathcal{C}}$, and therefore $L\left(\tilde{\mu}_{\text {sup }} ; \beta\right)^{\mathcal{C}}$ is an ideal of $X$.

The following example shows that the converse of Theorem 2 is not true, that is, there exists a hyper structure $(X, \tilde{\mu})$ over $(X, *, 0)$ such that

(1) $(X, \tilde{\mu})$ is not a $(2,3)$-hyperfuzzy ideal of $(X, *, 0)$,

(2) The nonempty sets $U\left(\tilde{\mu}_{\text {inf }} ; \alpha\right)^{c}$ and $L\left(\tilde{\mu}_{\text {sup }} ; \beta\right)^{c}$ are ideals of $(X, *, 0)$ for all $\alpha, \beta \in[0,1]$.

Example 3. Consider a BCI-algebra $X=\{0,1, a, b, c\}$ with the binary operation $*$ which is given in Table 3 (see [8]).

Table 3. Cayley table for the binary operation " $*$ ".

\begin{tabular}{llllll}
\hline$*$ & $\mathbf{0}$ & $\mathbf{1}$ & $\boldsymbol{a}$ & $\boldsymbol{b}$ & $\boldsymbol{c}$ \\
\hline 0 & 0 & 0 & $a$ & $b$ & $c$ \\
1 & 1 & 0 & $a$ & $b$ & $c$ \\
$a$ & $a$ & $a$ & 0 & $c$ & $b$ \\
$b$ & $b$ & $b$ & $c$ & 0 & $a$ \\
$c$ & $c$ & $c$ & $b$ & $a$ & 0 \\
\hline
\end{tabular}

Let $(X, \tilde{\mu})$ be a hyper structure over $(X, *, 0)$ in which $\tilde{\mu}$ is given as follows:

$$
\tilde{\mu}: X \rightarrow \tilde{\mathcal{P}}([0,1]), x \mapsto \begin{cases}{[0.23,0.85)} & \text { if } x=0, \\ (0.43,0.83] & \text { if } x=1, \\ {[0.53,0.73]} & \text { if } x=a, \\ (0.63,0.73] & \text { if } x=b, \\ {[0.63,0.75)} & \text { if } x=c .\end{cases}
$$

Then

$$
U\left(\tilde{\mu}_{\text {inf }} ; \alpha\right)^{c}= \begin{cases}\varnothing & \text { if } \alpha \in[0,0.23] \\ \{0\} & \text { if } \alpha \in(0.23,0.43] \\ \{0,1\} & \text { if } \alpha \in(0.43,0.53] \\ \{0,1, a\} & \text { if } \alpha \in(0.53,0.63] \\ X & \text { if } \alpha \in(0.63,1.0]\end{cases}
$$


and

$$
L\left(\tilde{\mu}_{\text {sup }} ; \beta\right)^{c}= \begin{cases}\varnothing & \text { if } \beta \in(0.85,1.0] \\ \{0\} & \text { if } \beta \in(0.83,0.85] \\ \{0,1\} & \text { if } \beta \in(0.75,0.83] \\ \{0,1, c\} & \text { if } \beta \in(0.73,0.75] \\ X & \text { if } \beta \in(0,0.73] .\end{cases}
$$

Hence the nonempty sets $U\left(\tilde{\mu}_{\text {inf }} ; \alpha\right)^{c}$ and $L\left(\tilde{\mu}_{\text {sup }} ; \beta\right)^{c}$ are ideals of $(X, *, 0)$ for all $\alpha, \beta \in[0,1]$. But $(X, \tilde{\mu})$ is not a (2,3)-hyperfuzzy ideal of $(X, *, 0)$ since

$$
\tilde{\mu}_{\text {inf }}(c)=0.63>0.53=\min \left\{\tilde{\mu}_{\text {inf }}(c * a), \tilde{\mu}_{\text {inf }}(a)\right\}
$$

and/or

$$
\tilde{\mu}_{\text {sup }}(a)=0.73<0.75=\max \left\{\tilde{\mu}_{\text {sup }}(a * c), \tilde{\mu}_{\text {inf }}(c)\right\} .
$$

Using the similar way to the proof of Theorem 2, we have the following theorem.

Theorem 3. (1) If a hyper structure $(X, \tilde{\mu})$ over $(X, *, 0)$ is a $(2,2)$-hyperfuzzy ideal of $(X, *, 0)$, then the sets $U\left(\tilde{\mu}_{\text {inf }} ; \alpha\right)^{c}$ and $U\left(\tilde{\mu}_{\text {sup }} ; \beta\right)^{c}$ are either empty or ideals of $(X, *, 0)$ for all $\alpha, \beta \in[0,1]$.

(2) If a hyper structure $(X, \tilde{\mu})$ over $(X, *, 0)$ is a $(3,2)$-hyperfuzzy ideal of $(X, *, 0)$, then the sets $L\left(\tilde{\mu}_{\text {inf }} ; \alpha\right)^{c}$ and $U\left(\tilde{\mu}_{\text {sup }} ; \beta\right)^{c}$ are either empty or ideals of $(X, *, 0)$ for all $\alpha, \beta \in[0,1]$.

(3) If a hyper structure $(X, \tilde{\mu})$ over $(X, *, 0)$ is a $(3,3)$-hyperfuzzy ideal of $(X, *, 0)$, then the sets $L\left(\tilde{\mu}_{\text {inf }} ; \alpha\right)^{c}$ and $L\left(\tilde{\mu}_{\text {sup }} ; \beta\right)^{c}$ are either empty or ideals of $(X, *, 0)$ for all $\alpha, \beta \in[0,1]$.

Using the similar way to the proof of Theorems 1 and 2, we have the following theorem.

Theorem 4. (1) If a hyper structure $(X, \tilde{\mu})$ over $(X, *, 0)$ is a $(1,2)$-hyperfuzzy ideal of $(X, *, 0)$, then the sets $U\left(\tilde{\mu}_{\mathrm{inf}} ; \alpha\right)$ and $U\left(\tilde{\mu}_{\text {sup }} ; \beta\right)^{c}$ are either empty or ideals of $(X, *, 0)$ for all $\alpha, \beta \in[0,1]$.

(2) If a hyper structure $(X, \tilde{\mu})$ over $(X, *, 0)$ is a $(1,3)$-hyperfuzzy ideal of $(X, *, 0)$, then the sets $U\left(\tilde{\mu}_{\text {inf }} ; \alpha\right)$ and $L\left(\tilde{\mu}_{\text {sup }} ; \beta\right)^{c}$ are either empty or ideals of $(X, *, 0)$ for all $\alpha, \beta \in[0,1]$.

(3) If a hyper structure $(X, \tilde{\mu})$ over $(X, *, 0)$ is a $(2,1)$-hyperfuzzy ideal of $(X, *, 0)$, then the sets $U\left(\tilde{\mu}_{\text {inf }} ; \alpha\right)^{c}$ and $U\left(\tilde{\mu}_{\text {sup }} ; \beta\right)$ are either empty or ideals of $(X, *, 0)$ for all $\alpha, \beta \in[0,1]$.

(4) If a hyper structure $(X, \tilde{\mu})$ over $(X, *, 0)$ is a $(3,1)$-hyperfuzzy ideal of $(X, *, 0)$, then the sets $L\left(\tilde{\mu}_{\mathrm{inf}} ; \alpha\right)^{c}$ and $U\left(\tilde{\mu}_{\text {sup }} ; \beta\right)$ are either empty or ideals of $(X, *, 0)$ for all $\alpha, \beta \in[0,1]$.

(5) If a hyper structure $(X, \tilde{\mu})$ over $(X, *, 0)$ is a $(2,4)$-hyperfuzzy ideal of $(X, *, 0)$, then the sets $U\left(\tilde{\mu}_{\text {inf }} ; \alpha\right)^{c}$ and $L\left(\tilde{\mu}_{\text {sup }} ; \beta\right)$ are either empty or ideals of $(X, *, 0)$ for all $\alpha, \beta \in[0,1]$.

(6) If a hyper structure $(X, \tilde{\mu})$ over $(X, *, 0)$ is a $(3,4)$-hyperfuzzy ideal of $(X, *, 0)$, then the sets $L\left(\tilde{\mu}_{\mathrm{inf}} ; \alpha\right)^{c}$ and $L\left(\tilde{\mu}_{\mathrm{sup}} ; \beta\right)$ are either empty or ideals of $(X, *, 0)$ for all $\alpha, \beta \in[0,1]$.

(7) If a hyper structure $(X, \tilde{\mu})$ over $(X, *, 0)$ is a $(4,2)$-hyperfuzzy ideal of $(X, *, 0)$, then the sets $L\left(\tilde{\mu}_{\text {inf }} ; \alpha\right)$ and $U\left(\tilde{\mu}_{\text {sup }} ; \beta\right)^{c}$ are either empty or ideals of $(X, *, 0)$ for all $\alpha, \beta \in[0,1]$.

(8) If a hyper structure $(X, \tilde{\mu})$ over $(X, *, 0)$ is a $(4,3)$-hyperfuzzy ideal of $(X, *, 0)$, then the sets $L\left(\tilde{\mu}_{\text {inf }} ; \alpha\right)$ and $L\left(\tilde{\mu}_{\text {sup }} ; \beta\right)^{c}$ are either empty or ideals of $(X, *, 0)$ for all $\alpha, \beta \in[0,1]$.

\section{Relations between Hyperfuzzy Ideals and Hyperfuzzy Subalgebras}

Theorem 5. Let $(X, *, 0) \in \mathcal{B}_{K}(X)$. For any $i, j \in\{1,4\}$, every $(i, j)$-hyperfuzzy ideal is an $(i, j)$-hyperfuzzy subalgebra. 
Proof. Let $(X, *, 0) \in \mathcal{B}_{K}(X)$ and let $(X, \tilde{\mu})$ be a $(1,4)$-hyperfuzzy ideal of $(X, *, 0)$. Then $\left(X, \tilde{\mu}_{\text {inf }}\right)$ is a 1-fuzzy ideal of $(X, *, 0)$ and $\left(X, \tilde{\mu}_{\text {sup }}\right)$ is a 4-fuzzy ideal of $(X, *, 0)$. Since $x * y \leq x$ for all $x, y \in X$, it follows from Proposition 1, (8) and (12) that

$$
\begin{aligned}
& \tilde{\mu}_{\text {inf }}(x * y) \geq \tilde{\mu}_{\text {inf }}(x) \geq \min \left\{\tilde{\mu}_{\text {inf }}(x * y), \tilde{\mu}_{\text {inf }}(y)\right\} \geq \min \left\{\tilde{\mu}_{\text {inf }}(x), \tilde{\mu}_{\text {inf }}(y)\right\} \\
& \tilde{\mu}_{\text {sup }}(x * y) \leq \tilde{\mu}_{\text {sup }}(x) \leq \max \left\{\tilde{\mu}_{\text {sup }}(x * y), \tilde{\mu}_{\text {sup }}(y)\right\} \leq \max \left\{\tilde{\mu}_{\text {sup }}(x), \tilde{\mu}_{\text {sup }}(y)\right\}
\end{aligned}
$$

for all $x, y \in X$. Therefore $(X, \tilde{\mu})$ is a $(1,4)$-hyperfuzzy subalgebra of $(X, *, 0)$. Similarly, we can prove the result for $(i, j) \in\{(1,1),(4,1),(4,4)\}$.

The converse of Theorem 5 is not true for $(i, j)=(1,4)$ as seen in the following example.

Example 4. Consider a $B C K$-algebra $X=\{0, a, b, c\}$ with the binary operation $*$ which is given in Table 4 (see [8]).

Table 4. Cayley table for the binary operation " $*$ ".

\begin{tabular}{lllll}
\hline$*$ & $\mathbf{0}$ & $\boldsymbol{a}$ & $\boldsymbol{b}$ & $\boldsymbol{c}$ \\
\hline 0 & 0 & 0 & 0 & 0 \\
$a$ & $a$ & 0 & 0 & $a$ \\
$b$ & $b$ & $a$ & 0 & $b$ \\
$c$ & $c$ & $c$ & $c$ & 0 \\
\hline
\end{tabular}

Let $(X, \tilde{\mu})$ be a hyper structure over $(X, *, 0)$ in which $\tilde{\mu}$ is given as follows:

$$
\tilde{\mu}: X \rightarrow \tilde{\mathcal{P}}([0,1]), x \mapsto \begin{cases}{[0.4,0.7]} & \text { if } x=0, \\ (0.4,0.7] & \text { if } x=a, \\ {[0.2,0.9]} & \text { if } x=b, \\ {[0.4,0.5) \cup(0.5,0.7)} & \text { if } x=c .\end{cases}
$$

It is routine to verify that $(X, \tilde{\mu})$ is a $(1,4)$-hyperfuzzy subalgebra of $(X, *, 0)$. But it is not a $(1,4)$-hyperfuzzy ideal of $(X, *, 0)$ since $\tilde{\mu}_{\text {sup }}(b)=0.9>0.7=\max \left\{\tilde{\mu}_{\text {sup }}(b * a), \tilde{\mu}_{\text {sup }}(a)\right\}$.

Example 5. Let $X=\{0,1,2,3,4\}$ be the BCK-algebra in Example 1. Let $(X, \tilde{\mu})$ be a hyper structure over $(X, *, 0)$ in which $\tilde{\mu}$ is given as follows:

$$
\tilde{\mu}: X \rightarrow \tilde{\mathcal{P}}([0,1]), x \mapsto \begin{cases}{[0.5,0.9)} & \text { if } x=0, \\ (0.4,0.6) \cup(0.6,0.8] & \text { if } x=1, \\ {[0.3,0.5]} & \text { if } x=2, \\ {[0.2,0.4) \cup(0.5,0.6]} & \text { if } x=3, \\ {[0.2,0.5]} & \text { if } x=4 .\end{cases}
$$

Then $(X, \tilde{\mu})$ is a $(1,1)$-hyperfuzzy subalgebra of $(X, *, 0)$. Since

$$
\tilde{\mu}_{\text {sup }}(4)=0.5<0.6=\min \left\{\tilde{\mu}_{\text {sup }}(4 * 3), \tilde{\mu}_{\text {sup }}(3)\right\},
$$

$\left(X, \tilde{\mu}_{\text {sup }}\right)$ is not a 1-fuzzy ideal of $X$. Hence $(X, \tilde{\mu})$ is not a $(1,1)$-hyperfuzzy ideal of $(X, *, 0)$.

Example 6. Consider a $B C K$-algebra $X=\{0, a, b, c\}$ with the binary operation $*$ which is given in Table 5 (see [8]). 
Table 5. Cayley table for the binary operation " $*$ ".

\begin{tabular}{lllll}
\hline$*$ & $\mathbf{0}$ & $\boldsymbol{a}$ & $\boldsymbol{b}$ & $\boldsymbol{c}$ \\
\hline 0 & 0 & 0 & 0 & 0 \\
$a$ & $a$ & 0 & 0 & 0 \\
$b$ & $b$ & $a$ & 0 & $a$ \\
$c$ & $c$ & $c$ & $c$ & 0 \\
\hline
\end{tabular}

(1) Let $(X, \tilde{\mu})$ be a hyper structure over $(X, *, 0)$ in which $\tilde{\mu}$ is given as follows:

$$
\tilde{\mu}: X \rightarrow \tilde{\mathcal{P}}([0,1]), x \mapsto\left\{\begin{array}{cl}
{[0.3,0.9]} & \text { if } x=0, \\
{[0.5,0.8]} & \text { if } x=a, \\
{[0.4,0.5]} & \text { if } x=b, \\
{[0.6,0.7)} & \text { if } x=c .
\end{array}\right.
$$

Then $(X, \tilde{\mu})$ is a $(4,1)$-hyperfuzzy subalgebra of $(X, *, 0)$. Since

$$
\tilde{\mu}_{\mathrm{inf}}(1)=0.5>0.4=\max \left\{\tilde{\mu}_{\mathrm{inf}}(1 * 2), \tilde{\mu}_{\mathrm{inf}}(2)\right\}
$$

and/or

$$
\tilde{\mu}_{\text {sup }}(2)=0.5<0.8=\min \left\{\tilde{\mu}_{\text {sup }}(2 * 1), \tilde{\mu}_{\text {sup }}(1)\right\},
$$

$\left(X, \tilde{\mu}_{\text {inf }}\right)$ is not a 4 -fuzzy ideal of $(X, *, 0)$ and/or $\left(X, \tilde{\mu}_{\text {sup }}\right)$ is not a 1 -fuzzy ideal of $(X, *, 0)$. Therefore $(X, \tilde{\mu})$ is not a $(4,1)$-hyperfuzzy ideal of $(X, *, 0)$.

(2) Let $(X, \tilde{\mu})$ be a hyper structure over $(X, *, 0)$ in which $\tilde{\mu}$ is given as follows:

$$
\tilde{\mu}: X \rightarrow \tilde{\mathcal{P}}([0,1]), x \mapsto\left\{\begin{array}{cc}
{[0.3,0.5]} & \text { if } x=0, \\
{[0.5,0.7]} & \text { if } x=a, \\
{[0.4,0.7)} & \text { if } x=b, \\
{[0.6,0.8]} & \text { if } x=c .
\end{array}\right.
$$

Then $(X, \tilde{\mu})$ is a $(4,4)$-hyperfuzzy subalgebra of $(X, *, 0)$ and $\left(X, \tilde{\mu}_{\text {sup }}\right)$ is a 4-fuzzy ideal of $(X, *, 0)$. $B u t\left(X, \tilde{\mu}_{\text {inf }}\right)$ is not a 4 -fuzzy ideal of $(X, *, 0)$ since

$$
\tilde{\mu}_{\text {inf }}(1)=0.5>0.4=\max \left\{\tilde{\mu}_{\text {inf }}(1 * 2), \tilde{\mu}_{\text {inf }}(2)\right\} .
$$

Hence $(X, \tilde{\mu})$ is not a $(4,4)$-hyperfuzzy ideal of $(X, *, 0)$.

We provide conditions for a $(1,4)$-hyperfuzzy subalgebra to be a $(1,4)$-hyperfuzzy ideal.

Theorem 6. For any $(X, *, 0) \in \mathcal{B}_{K}(X)$, if a $(1,4)$-hyperfuzzy subalgebra $(X, \tilde{\mu})$ of $(X, *, 0)$ satisfies the condition (22), then $(X, \tilde{\mu})$ is a $(1,4)$-hyperfuzzy ideal of $(X, *, 0)$.

Proof. Let $(X, *, 0) \in \mathcal{B}_{K}(X)$ and let $(X, \tilde{\mu})$ be a $(1,4)$-hyperfuzzy subalgebra of $(X, *, 0)$ that satisfies (22). Since $0 * x \leq x$ for all $x \in X$, we have $\tilde{\mu}_{\text {inf }}(0) \geq \tilde{\mu}_{\text {inf }}(x)$ and $\tilde{\mu}_{\text {sup }}(0) \leq \tilde{\mu}_{\text {sup }}(x)$ for all $x \in X$ by (22). Since $x *(x * y) \leq y$ for all $x, y \in X$, it follows from (22) that

$$
\tilde{\mu}_{\text {inf }}(x) \geq \min \left\{\tilde{\mu}_{\text {inf }}(x * y), \tilde{\mu}_{\text {inf }}(y)\right\}
$$


and

$$
\tilde{\mu}_{\text {sup }}(x) \leq \max \left\{\tilde{\mu}_{\text {sup }}(x * y), \tilde{\mu}_{\text {sup }}(y)\right\}
$$

for all $x, y \in X$. Hence $(X, \tilde{\mu})$ is a $(1,4)$-hyperfuzzy ideal of $(X, *, 0)$.

Using the similar way to the proof of Theorem 6, we have the following theorem.

Theorem 7. For any $(X, *, 0) \in \mathcal{B}_{K}(X)$, we have the following assertions.

(1) If $(X, \tilde{\mu})$ is a $(1,1)$-hyperfuzzy subalgebra of $(X, *, 0)$ which satisfies the condition (23), then $(X, \tilde{\mu})$ is a (1,1)-hyperfuzzy ideal of $(X, *, 0)$.

(2) If $(X, \tilde{\mu})$ is a $(4,1)$-hyperfuzzy subalgebra of $(X, *, 0)$ which satisfies the condition (24), then $(X, \tilde{\mu})$ is a $(4,1)$-hyperfuzzy ideal of $(X, *, 0)$.

(3) If $(X, \tilde{\mu})$ is a $(4,4)$-hyperfuzzy subalgebra of $(X, *, 0)$ which satisfies the condition (25), then $(X, \tilde{\mu})$ is a $(4,4)$-hyperfuzzy ideal of $(X, *, 0)$.

Theorem 8. For any $(X, *, 0) \in \mathcal{B}_{K}(X)$ and $i, j \in\{2,3\}$, every $(i, j)$-hyperfuzzy ideal is an $(i, j)$-hyperfuzzy subalgebra.

Proof. Let $(X, *, 0) \in \mathcal{B}_{K}(X)$ and let $(X, \tilde{\mu})$ be a $(2,3)$-hyperfuzzy ideal of $(X, *, 0)$. Then $\left(X, \tilde{\mu}_{\text {inf }}\right)$ is a 2-fuzzy ideal of $(X, *, 0)$ and $\left(X, \tilde{\mu}_{\text {sup }}\right)$ is a 3-fuzzy ideal of $(X, *, 0)$. Since $x * y \leq x$ for all $x, y \in X$, we have

$$
\begin{aligned}
& \tilde{\mu}_{\text {inf }}(x * y)=\tilde{\mu}_{\text {inf }}(0) \leq \min \left\{\tilde{\mu}_{\text {inf }}(x), \tilde{\mu}_{\text {inf }}(y)\right\} \\
& \tilde{\mu}_{\text {sup }}(x * y)=\tilde{\mu}_{\text {sup }}(0) \geq \max \left\{\tilde{\mu}_{\text {sup }}(x), \tilde{\mu}_{\text {sup }}(y)\right\}
\end{aligned}
$$

for all $x, y \in X$ by Proposition 2, (9) and (7). Hence $(X, \tilde{\mu})$ is a (2,3)-hyperfuzzy subalgebra of $(X, *, 0)$. Similarly, we can prove it for $(i, j) \in\{(2,2),(3,2),(3,3)\}$.

Using the similar way to the proof of Theorems 5 and 8, we have the following theorem.

Theorem 9. For any $(X, *, 0) \in \mathcal{B}_{K}(X)$, every $(i, j)$-hyperfuzzy ideal is an $(i, j)$-hyperfuzzy subalgebra for $(i, j) \in\{(1,2),(1,3),(2,1),(2,4),(3,1),(3,4),(4,2),(4,3)\}$.

\section{Conclusions}

In the paper [2], Ghosh and Samanta have introduced the concept of hyperfuzzy sets as a generalization of fuzzy sets and interval-valued fuzzy sets, and have presented an application of hyperfuzzy sets in group theory. Jun et al. [6] have applied the hyperfuzzy sets to $B C K / B C I$-algebras. In this article, we have discussed ideal theory in $B C K / B C I$-algebras by using the hyperfuzzy sets, and have introduced the notion of hyperfuzzy ideals in $B C K / B C I$-algebras, and have investigate several properties. We have considered characterizations of hyperfuzzy ideals, and have discussed relations between hyperfuzzy subalgebras and hyperfuzzy ideals. We have provided conditions for hyperfuzzy subalgebras to be hyperfuzzy ideals. Recently, many kinds of fuzzy sets have several applications to deal with uncertainties from our different kinds of daily life problems, in particular, for solving decision making problems (see [9-13]). In the future, we shall extend our proposed approach to some decision making problem under the field of fuzzy cluster analysis, decision-making, uncertain programming and mathematical programming [9]. Moreover, we will apply the notions and results in this manuscript to related algebraic structures, for example, $M V$-algebras, $B L$-algebras, $M T L$-algebras, EQ-algebras, effect algebras, and so on.

Acknowledgments: The authors wish to thank the anonymous reviewers for their valuable suggestions. The first author, S. Z. Song, was supported by Basic Science Research Program through the National Research Foundation of Korea (NRF) funded by the Ministry of Education (No. 2016R1D1A1B02006812). 
Author Contributions: All authors contributed equally and significantly to the study and preparation of the article. They have read and approved the final manuscript.

Conflicts of Interest: The authors declare no conflict of interest.

\section{References}

1. Zadeh, L.A. Fuzzy sets. Inf. Control. 1965, 8, 338-353.

2. Ghosh, J.; Samanta, T.K. Hyperfuzzy sets and hyperfuzzy group. Int. J. Adv. Sci. Technol. 2012, 41, $27-37$.

3. Imai, Y.; Iséki, K. On axiom systems of propositional calculi. Proc. Jpn. Acad. Ser. A Math. Sci. 1966, 42, 19-21.

4. Iséki, K. An algebra related with a propositional calculus. Proc. Jpn. Acad. Ser. A Math. Sci. 1966, 42, $26-29$.

5. Iseki, K.; Tanaka, S. An introduction to the theory of BCK-algebras. Math. Jpn. 1978, 23, 1-26

6. Jun, Y.B.; Hur, K.; Lee, K.J. Hyperfuzzy subalgebras of BCK/BCI-algebras. Ann. Fuzzy Math. Inf. 2017, in press.

7. Huang, Y.S. BCI-Algebra; Science Press: Beijing, China, 2006.

8. Meng, J.; Jun, Y.B. BCK-Algebras; Kyungmoon Sa Co.: Seoul, Korea, 1994.

9. Garg, H. A robust ranking method for intuitionistic multiplicative sets under crisp, interval environments and its applications. IEEE Trans. Emerg. Top. Comput. Intell. 2017, 1, 366-374.

10. Feng, F.; Jun, Y.B.; Liu, X.; Li, L. An adjustable approach to fuzzy soft set based decision making. J. Comput. Appl. Math. 2010, 234, 10-20.

11. Xia, M.; Xu, Z. Hesitant fuzzy information aggregation in decision making. Int. J. Approx. Reason. 2011, 52, 395-407.

12. Tang, H. Decision making based on interval-valued intuitionistic fuzzy soft sets and its algorithm. J. Comput. Anal. Appl. 2017, 23, 119-131.

13. Wei, G.; Alsaadi, F.E.; Hayat, T.; Alsaedi, A. Hesitant bipolar fuzzy aggregation operators in multiple attribute decision making. J. Intell. Fuzzy Syst. 2017, 33, 1119-1128.

(C) 2017 by the authors. Licensee MDPI, Basel, Switzerland. This article is an open access article distributed under the terms and conditions of the Creative Commons Attribution (CC BY) license (http:/ / creativecommons.org/licenses/by/4.0/). 